\title{
Reproducción de la sardina, Sardinella aurita (Cupleiformes: Clupeidae) del sureste de la Isla de Margarita, Venezuela
}

\author{
Juan Gassman ${ }^{1}$, Nora Eslava² \& Leo W. González ${ }^{2}$ \\ 1. Escuela de Ciencias Aplicadas del Mar, Universidad de Oriente, Núcleo de Nueva Esparta, Boca del Río, Isla de \\ Margarita, Venezuela; gassman_udo@yahoo.com \\ 2. Área de Biología y Recursos Pesqueros, Instituto de Investigaciones Científicas, Universidad de Oriente, Núcleo de \\ Nueva Esparta. A.P. 749, Porlamar, Venezuela; leonora@movistar.net.ve; walter@mda.cinvestav.mx
}

Recibido 24-III-2008. Corregido 30-VI-2008. Aceptado 31-VII-2008.

\begin{abstract}
Reproduction of the Spanish sardine, Sardinella aurita (Clupeiformes: Clupeidae) from the south-eastern area of Margarita Island, Venezuela. Sex ratio, sexual maturity, fecundity and time of spawning of the Spanish sardine (Sardinella aurita) from the south-eastern area of Margarita Island were determined through monthly samplings of commercial fisheries, from January 2004 to April 2005. A total of 3736 individuals were examined. Sex ratio was $55.47 \%$ females and $44.53 \%$ males, with a $1: 1.25$ sexual proportion $\left(\chi^{2}=44.454, \mathrm{p}<0.05\right)$. Length at first sexual maturity $\left(\mathrm{L}_{50}\right)$ was $20 \mathrm{~cm}$. Spawning of the species was confirmed to be partial and continuous throughout the year, with two peaks of intensity during the first and last quarters. Absolute fecundity ranged from 10530 to 83779 oocytes and it was proportional to body length $(\mathrm{F}=-100900+6696.2 * \mathrm{~L})$ and to body weight $(\mathrm{F}=13327+5666.3 * \mathrm{P})$. Relative fecundity ranged from 149 to 1020 oocytes/g and also was proportional to body length and weight. We concluded that the Spanish sardine exhibits two peaks of spawning activity per year with a very fluctuating partial fecundity which could possibly be explained by environmental variability. Rev. Biol. Trop. 56 (4): 1813-1824. Epub 2008 December 12.
\end{abstract}

Key words: Sardinella aurita, reproductive, fecundity, Margarita Island, Venezuela.

La sardina, Sardinella aurita Valenciennes, 1847 es una especie pelágica costanera, que forma cardúmenes muy compactos (Cervigón 1991). Se distribuye desde Estados Unidos hasta Brasil; reportándose también en el Mar Mediterráneo y en el Pacífico oeste (Fischer 1977). En Venezuela se localiza principalmente en la región oriental, en zonas muy cercanas a la costa, donde el evento de surgencia producido por los vientos locales es más intenso, lo cual determina una alta productividad biológica (Etchevers 1974, Mendoza 1990).

La sardina es uno de los recursos pesqueros más importantes para Venezuela, pues sostiene una de las pesquerías más relevantes, debido a los grandes volúmenes de captura y a las numerosas fuentes de trabajo que se genera por su extracción, procesamiento y comercialización
(Ramírez y Huq 1986, Mendoza 1990, Guzmán y Penott 1999, González 2006). Prospecciones hidroacústicas han estimado una biomasa total entre 800000 a 1000000 t (Mendoza 1990). Sin embargo, el aparente crecimiento sostenido de la pesquería se ha visto afectado con la drástica disminución de las capturas en los tres últimos años.

El conocimiento sobre los aspectos reproductivos es de gran utilidad para la comprensión de la biología básica de un recurso, siendo la fecundidad el factor que influye en la abundancia y recuperación de una población (Parker 1985, Tresierra y Culquichicón 1993). En Venezuela se han realizado estudios sobre la biología reproductiva (Figuera 1967, D Suze 1981, Reyes 1981, Ramírez y Huq 1986, Frèon et al. 1997, Guzmán et al. 1999, Barrios 
2002), pero poco se conoce sobre estimaciones de la población reproductora, fecundidad y stock-reclutamiento; conscientes que $S$. aurita, como todo clupeido, es sensible a los cambios ambientales y a la intensidad de pesca, motivo suficiente para ser evaluado regularmente y elaborar adecuados planes de manejo (González 1985, Guzmán y Penott 1999, González y Eslava 2000, González 2006). Ante esta realidad, se consideró oportuno determinar algunos aspectos reproductivos de la sardina del sureste de la Isla de Margarita, donde se ubican los caladeros más importantes de la región nororiental de Venezuela, con la finalidad de contribuir con la dinámica reproductiva y la administración de esta pesquería.

\section{MATERIALES Y MÉTODOS}

El presente estudio se basó en ejemplares obtenidos mensualmente de la pesca artesanal del sureste de la Isla de Margarita, Venezuela (Fig. 1), entre los meses de enero de 2004 y abril de 2005. A cada ejemplar se le determinó la longitud total (L) y el peso total (P). Posteriormente las gónadas fueron extraídas mediante disección abdominal, determinándose el sexo y la fase de madurez sexual de acuerdo a la escala de reproductores parciales modificada de Holden y Raitt (1975): Fase I (virgen), Fase II (en maduración o en recuperación), Fase III (maduro) y Fase IV (desovado). Posteriormente, se pesaron las gónadas de machos y hembras con una balanza de $0,1 \mathrm{~g}$ de precisión.

Para analizar si existían desviaciones significativas respecto a la proporción sexual 1:1 se aplicó la prueba Chi-cuadrado $\left(\chi^{2}=\mathrm{p}<\right.$ $0.05)$ con corrección de Yates para continuidad (Steel y Torrie 1985); y con el fin de verificar si la proporción sexual fue variable durante los meses de estudio, se empleó una prueba de heterogeneidad de la Chi-cuadrado (Steel y Torrie 1985). El índice de gónadas se obtuvo mediante lo anotado por Schaefer y Orange (1956): IG $=\mathrm{Pg} / \mathrm{L}^{3}$. Donde: IG es el índice de gónadas; Pg es el peso de las gónadas y L es la longitud total del pez. La talla media de madurez sexual de la población $\left(\mathrm{L}_{50}\right)$ se obtuvo estimando la fracción de ejemplares maduros en cada intervalo de talla, tanto para machos como para hembras, mediante la ecuación de la curva logística modificada de Hoydal et al. (1982), sugerida por Gaertner y Laloe (1986) y Palazón (2007): FM ${ }_{(\mathrm{L})}=1 / 1+\mathrm{e}^{(\mathrm{S} 1-\mathrm{S} 2 * \mathrm{~L})}$.

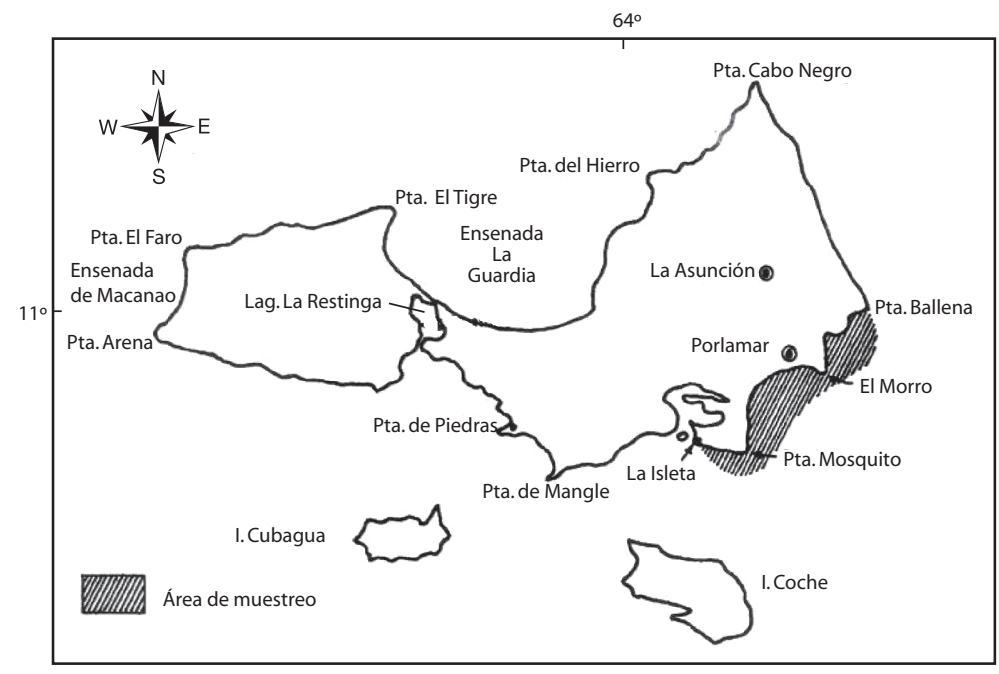

Fig. 1 Área de pesca de Sardinella aurita al sureste de la Isla de Margarita, Venezuela.

Fig. 1 Fishing area of Sardinella aurita in south-eastern of Margarita Island, Venezuela. 
Donde: $\mathrm{FM}_{(\mathrm{L})}$ es la proporción de individuos maduros a cada talla; $\mathrm{S}_{1}$ y $\mathrm{S}_{2}$ son constantes del modelo y $\mathrm{L}$ es la marca de clase de la longitud de referencia. Para calcular $\mathrm{S}_{1}$ y $\mathrm{S}_{2}$ se usó la ecuación de la regresión lineal: $\operatorname{Ln}\left(1 / \mathrm{F}_{(\mathrm{L})}-1\right)$ $=\mathrm{S}_{1}-\mathrm{S}_{2} * \mathrm{~L} . \mathrm{L}_{50}=\mathrm{S}_{1} / \mathrm{S}_{2}$. Donde: $\mathrm{F}_{(\mathrm{L})}$ es la fracción a cada talla; $\mathrm{L}$ es la marca de clase; $\mathrm{S}_{1}$ $=$ a y $\mathrm{S}_{2}=-$ b (Eslava 2007).

Los ovarios que se encontraron en fase de madurez sexual III, fueron conservados en líquido de Gilson modificado $(100 \mathrm{ml}$ de alcohol de $60^{\circ}, 800 \mathrm{ml}$ de agua, $15 \mathrm{ml}$ de ácido nítrico al 80\%, $18 \mathrm{ml}$ de ácido acético glacial y $20 \mathrm{~g}$ de cloruro de mercurio) durante no menos de 48 horas, siendo agitados eventualmente para desprender los óvulos del tejido ovárico.

El diámetro de los óvulos de hembras maduras de diferente longitud escogidos al azar, fue medido, bajo un microscopio binocular, utilizando una escala micrométrica adaptada a un ocular de 10X. Posteriormente, se realizó una distribución de frecuencia del diámetro de los óvulos, a fin de seleccionar el último grupo modal a considerar en la estimación de la fecundidad, de acuerdo a lo sugerido por Tresierra y Culquichicón (1993).

La fecundidad absoluta se calculó utilizando el método gravimétrico propuesto por Holden y Raitt (1975): F = n * Pt / Pn. Donde: $\mathrm{F}$ es la fecundidad; $\mathrm{n}$ es el número de óvulos maduros en la submuestra; Pt es el peso total de los óvulos y Pn es el peso de los óvulos de la submuestra. Las relaciones entre la fecundidad y la talla, y el peso, fueron estimadas por regresión lineal de modelo II según lo recomendado por Ricker (1973). La fecundidad relativa (FR) fue calculada mediante la ecuación sugerida por Tresierra y Culquichicón (1993): FR = F/ P. Donde: F es la fecundidad absoluta y P es el peso total $(\mathrm{g})$ de cada individuo.

\section{RESULTADOS}

Se analizaron 3736 ejemplares con tallas comprendidas entre 13.3 y $24.4 \mathrm{~cm}$ de longitud total y entre 18.0 y $118.51 \mathrm{~g}$ en peso. De ese total, 2057 fueron hembras (55.06\%), 1651 machos (44.19\%) y 28 de sexo indeterminado $(0,75 \%)$.
Proporción sexual: el porcentaje de machos y hembras de $S$. aurita varió a lo largo de todo el periodo de muestreo, y la proporción sexual fue 1:1 en la mayoría de los meses; sin embargo, para la totalidad del periodo de estudio la proporción se desvió de la igualdad y fue 1:1.25. En general, las hembras fueron más numerosas que los machos, excepto en septiembre y abril (Cuadro 1).

Proporción mensual de los estadios de madurez sexual: en la Fig. 2 se presenta la variación mensual de las fases de madurez sexual, donde la fase I (individuos inmaduros), estuvo levemente representada en los dos primeros trimestres del año, mientras que la fase II (en maduración) mostró mayor intensidad para el mismo lapso. La fase III (individuos maduros) varió a lo largo del año evidenciando dos periodos con valores altos, en el primer y último trimestre y la fase IV (individuos desovados) tuvo un comportamiento similar a la fase anterior.

Índice de gónadas: el índice de gónadas mostró variación a lo largo de todo el periodo de estudio, reconociéndose un desove continuo con dos periodos de relativa intensidad reproductiva, uno de mayor actividad que ocurre de enero a marzo con un valor máximo en este último mes, y otro de menor magnitud de octubre a diciembre con un valor máximo en el mes de noviembre (Fig. 3).

Talla de madurez sexual del $50 \%$ de la población: debido a que las constantes estimadas por el modelo logístico para machos $\left(\mathrm{S}_{1}=\right.$ 37.49 y $\left.\mathrm{S}_{2}=1.90\right)$ y hembras $\left(\mathrm{S}_{1}=32.46\right.$ y $\mathrm{S}_{2}$ $=1.60)$ fueron similares, se determinó una sola talla de primera madurez sexual del $50 \%$ de la población, donde $\mathrm{S}_{1}=35.64$ y $\mathrm{S}_{2}=1.7823$ permitieron estimar un $\mathrm{L}_{50}=20 \mathrm{~cm}$ de longitud total (Fig. 4).

Fecundidad: el diámetro de los óvulos osciló de 25 a $600 \mu$ y su distribución de frecuencia demostró la presencia de dos y tres grupos modales en ovarios maduros, siendo 
CUADRO 1

Proporción sexual mensual de S. aurita del sureste de la Isla de Margarita de enero 2004 a abril 2005

$\left(\chi^{2}\right.$ tabulado $\left.=3.841\right)$

TABLE 1

Monthly sex ratio of S. aurita of the south-eastern area of Margarita Island of January 2004 to April 2005 $\left(\chi^{2} t a b=3.841\right)$

$\begin{array}{lcccccc}\text { Mes/Año } & & \text { Machos } & \text { Hembras } & \text { Total } & \chi^{2} & \text { Significancia } \\ \text { Enero } & 2004 & 135 & 137 & 272 & 0.015 & p>0.05 \\ \text { Febrero } & 2004 & 138 & 157 & 295 & 1.224 & p>0.05 \\ \text { Marzo } & 2004 & 133 & 156 & 289 & 1.83 & p>0.05 \\ \text { Abril } & 2004 & 122 & 182 & 304 & 11.842 & p<0.05 \\ \text { Mayo } & 2004 & 102 & 130 & 232 & 3.379 & p>0.05 \\ \text { Junio } & 2004 & 59 & 109 & 168 & 14.881 & p<0.05 \\ \text { Julio } & 2004 & 82 & 161 & 243 & 25.683 & p<0.05 \\ \text { Agosto } & 2004 & 103 & 100 & 203 & 0.044 & p>0.05 \\ \text { Septiembre } & 2004 & 118 & 85 & 203 & 5.364 & p<0.05 \\ \text { Octubre } & 2004 & 89 & 115 & 204 & 3.314 & p>0.05 \\ \text { Noviembre } & 2004 & 91 & 124 & 215 & 5.065 & p<0.05 \\ \text { Diciembre } & 2004 & 75 & 137 & 212 & 18.132 & p<0.05 \\ \text { Enero } & 2005 & 90 & 114 & 204 & 2.823 & p>0.05 \\ \text { Febrero } & 2005 & 85 & 121 & 206 & 6.291 & p<0.05 \\ \text { Marzo } & 2005 & 102 & 105 & 207 & 0.043 & p>0.05 \\ \text { Abril } & 2005 & 127 & 124 & 251 & 0.036 & p>0.05 \\ \text { Total } & & 1651 & 2057 & 3708 & 44.454 & p<0.05\end{array}$

los óvulos de $350 \mu$ los más constantes, por lo que fueron considerados en la estimación de la fecundidad a partir de esa medida (Fig. 5). La fecundidad absoluta osciló desde 10530 hasta 83779 óvulos por hembra, con una media de 40596 ovocitos y una desviación estándar de \pm 16175 óvulos, presentando variaciones para una misma talla y peso (Cuadro 2). La fecundidad relativa fluctuó entre 149 a 1020 óvulos/g con una media de 519 óvulos/g y una desviación estándar de \pm 203 óvulos/g (Cuadro 2). Las relaciones entre la fecundidad y la talla (Fig. 6), y la fecundidad y el peso (Fig. 7) quedaron definidas por las ecuaciones de regresión lineal: $\mathrm{F}=-100900+6696.2 *$ L y $\mathrm{F}=13327$ $+5666.3 * \mathrm{P}$, respectivamente.

\section{DISCUSIÓN}

La proporción sexual fue diferente de la unidad, siendo las hembras más numerosas que los machos, lo cual concuerda con Heald y Griffiths (1967) para individuos del Golfo de Cariaco, Mendialdúa (2004) para el sureste de la isla de Margarita y Tsikliras y Antonopoulou (2006) para $S$. aurita del Mar Egeo. Sin embargo, discrepa de resultados obtenidos por Reyes (1981), Etchevers (1974), Ramírez y Huq (1986), Frèon et al. (1997) y Guzmán et al. (1998, 1999), quienes indican que para el nororiente de Venezuela la proporción sexual de la especie es muy cercana a 1 . Tales diferencias intraespecíficas pueden deberse a cambios 

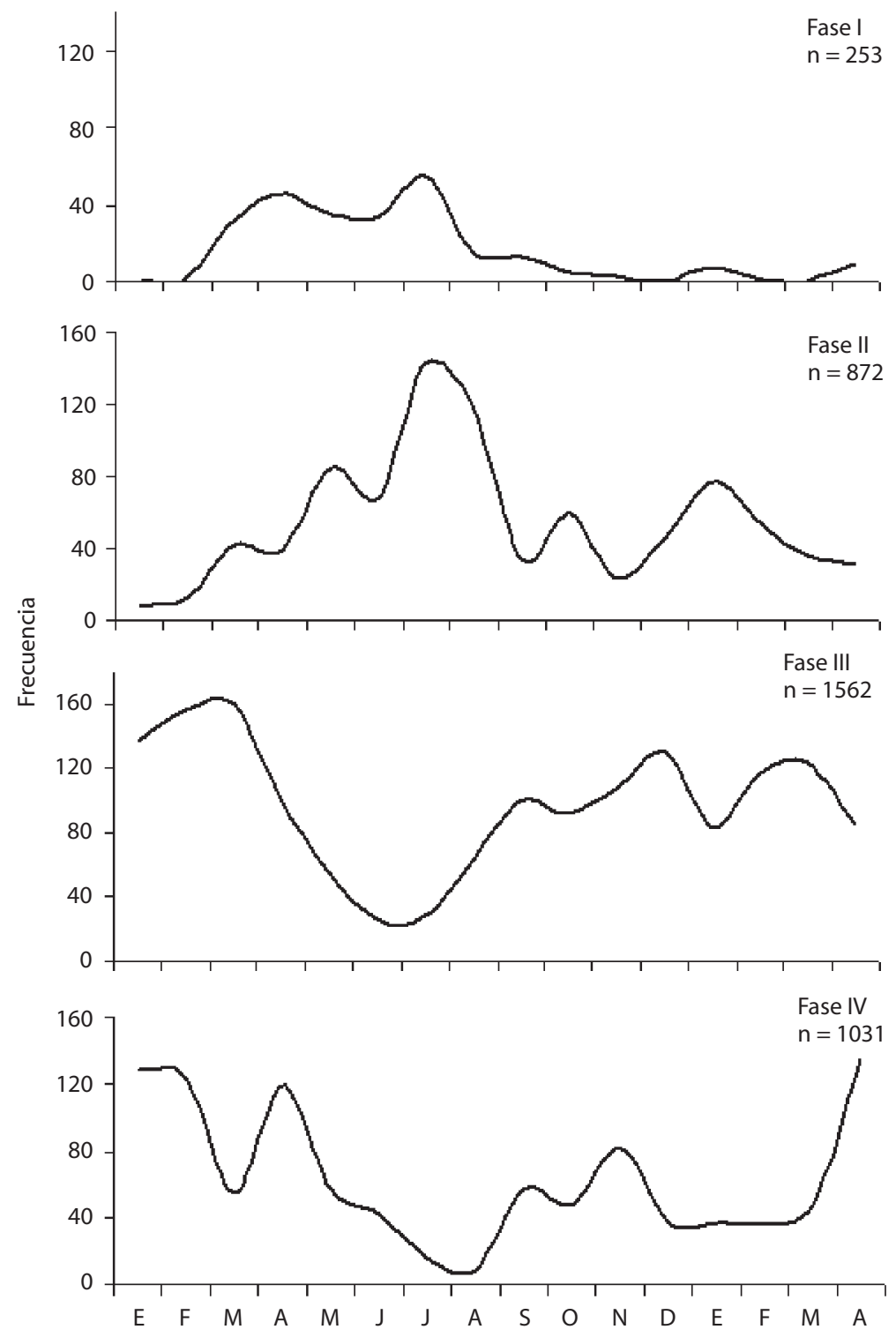

Meses (2004-2005)

Fig. 2 Variación mensual de la frecuencia absoluta de las fases de madurez sexual de Sardinella aurita del sureste de la Isla de Margarita de enero 2004 a abril 2005.

Fig. 2 Monthly variation of absolute frequency maturity stages of Sardinella aurita of the south-eastern area of Margarita Island of January 2004 to April 2005. 


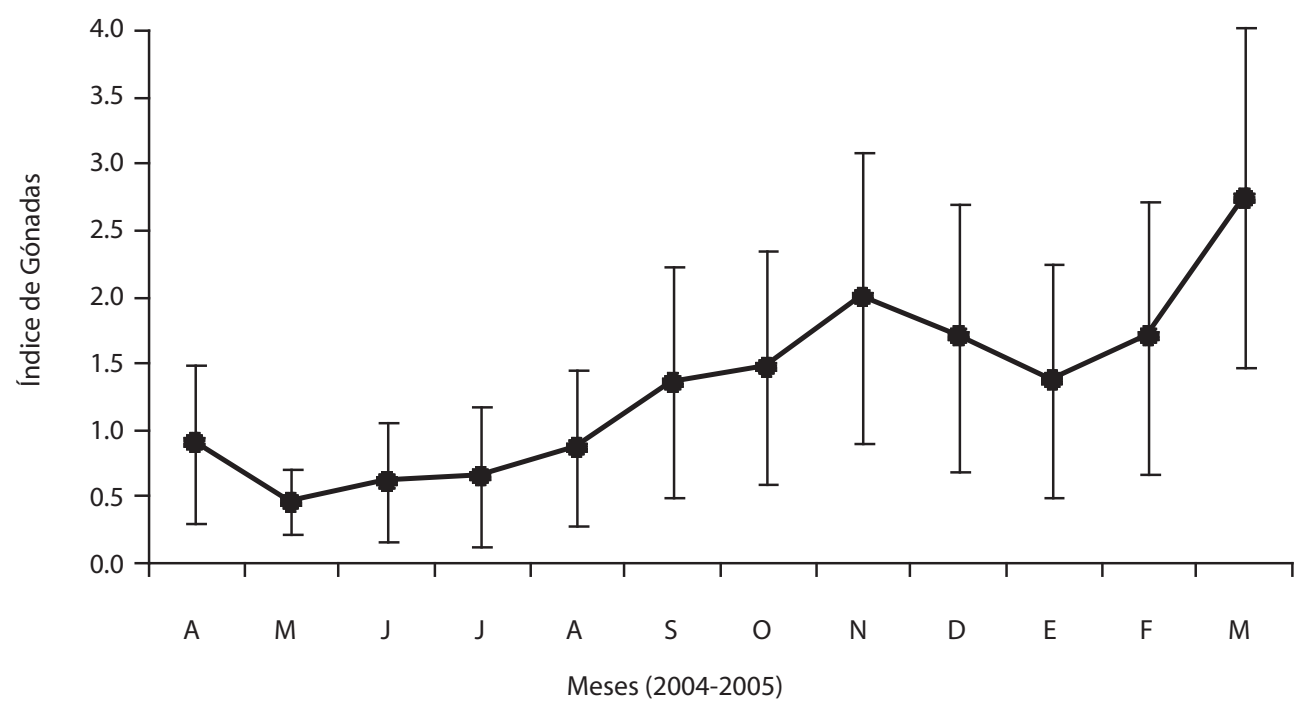

Fig. 3 Variación mensual de índice de gónadas de Sardinella aurita del sureste de la Isla de Margarita de abril 2004 a abril 2005.

Fig. 3 Monthly variation of the gonadosomatic index of Sardinella aurita of the south-eastern area of Margarita Island of April 2004 to April 2005.

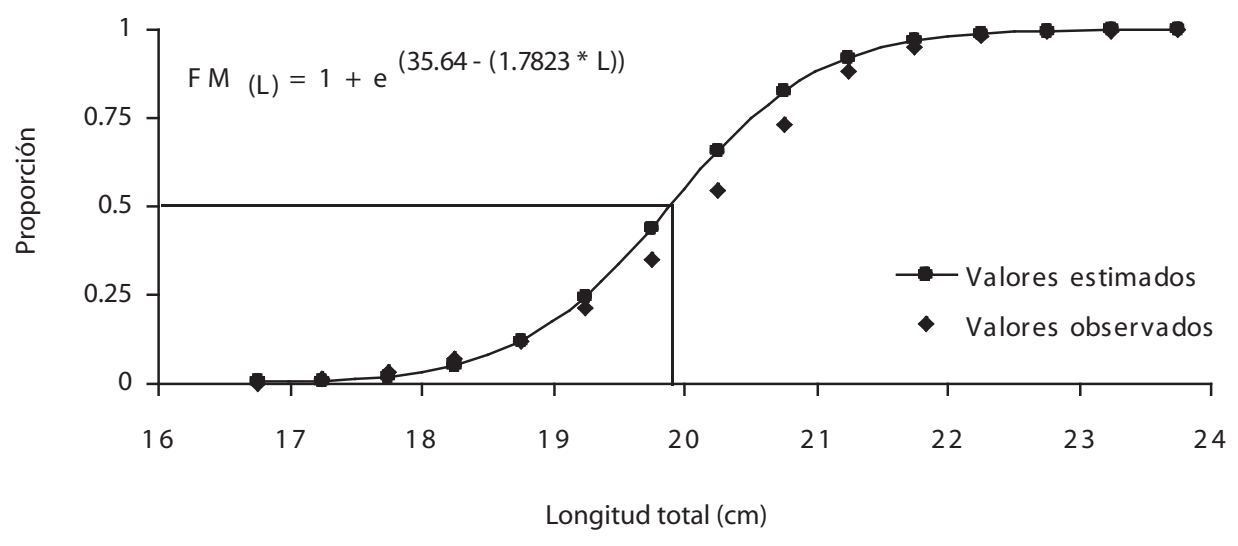

Fig. 4 Talla media de madurez sexual de la población $\left(\mathrm{L}_{50}\right)$ de Sardinella aurita del sureste de la Isla de Margarita.

Fig. 4 Sexual maturity curve and size at maturity from $50 \%$ of Sardinella aurita of the south-eastern area of Margarita Island. 

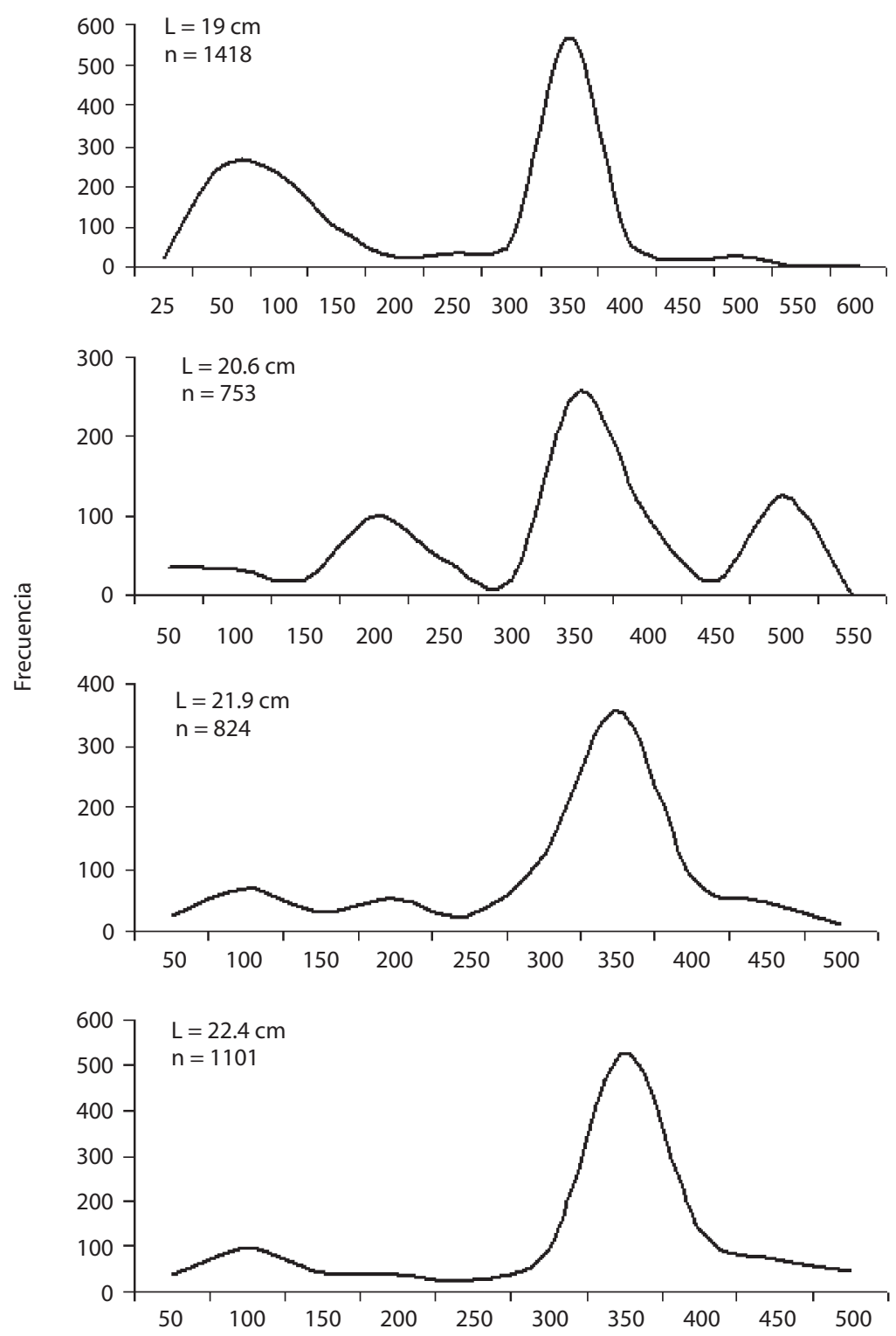

Diámetro de óvulos $(u)$

Fig. 5 Distribución de frecuencia del diámetro de óvulos en ovarios de hembras maduras de diferentes longitudes de Sardinella aurita del sureste de la Isla de Margarita

Fig. 5 Size distribution of oocytes diameters in the ovary of mature females of different length overalls of Sardinella aurita of the south-eastern area of Margarita Island. 
CUADRO 2

Valores máximos, mínimos y promedios de la fecundidad absoluta y relativa por intervalos de talla y peso de $\mathrm{S}$. aurita del sureste de la Isla de Margarita

TABLE 2

Values maximum, minimum and averages of the absolute and relative fecundity by intervals of length and weight of $\mathrm{S}$. aurita of the south-eastern area of Margarita Island

Fecundidad absoluta (óvulos)

$\begin{array}{cc}\text { Talla }(\mathrm{cm}) & \mathrm{n} \\ 18.5 & 1 \\ 19.0 & 4 \\ 19.5 & 1 \\ 20.0 & 8 \\ 20.5 & 10 \\ 21.0 & 14 \\ 21.5 & 6 \\ 22.0 & 8 \\ 22.5 & 1\end{array}$

Peso (g)

40
50
60
70
80
90
100

$$
\begin{gathered}
\text { Rango } \\
- \\
10530-38739 \\
- \\
15810-57528 \\
11640-79424 \\
24651-83779 \\
25920-60345 \\
30272-62567
\end{gathered}
$$

\section{1}

1

$$
\begin{gathered}
- \\
- \\
24714-57528 \\
10530-71698 \\
15810-83779 \\
25920-79424 \\
43430-60345
\end{gathered}
$$

\section{)}

10632

26569

36564

37596

41268

47103

40919

41861

44830

10632

26736

38623

38389

43125

43790

50647

Fecundidad relativa (óvulos/g)

$$
\text { DE }
$$

$$
+11819
$$$$
-
$$

$$
\pm 13761
$$$$
+21865
$$$$
+16237
$$

$$
\text { Rango }
$$$$
-
$$$$
149-622
$$$$
192-867
$$$$
+12758
$$$$
\pm 11426
$$$$
\begin{aligned}
& 192-867 \\
& 157-994
\end{aligned}
$$$$
312-1020
$$$$
263-591
$$$$
309-763
$$$$
\text { Media DE }
$$$$
-
$$$$
-
$$$$
225
$$$$
427
$$$$
+199
$$$$
597
$$$$
551
$$$$
535
$$$$
601
$$$$
\pm 272
$$$$
+196
$$$$
458
$$$$
\pm 125
$$$$
\pm 125
$$$$
444
$$$$
490
$$$$
\pm 141
$$

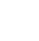

226

481

591

$+166$

$+11001$

$370-833$

524

$+225$

$+16252$

$149-994$

520

$\pm 245$

$\pm 20053$

$192-1020$

$\pm 16666$

$263-879$

468

$\pm 194$

$+7115$

$426-559$

461

$\pm 56$

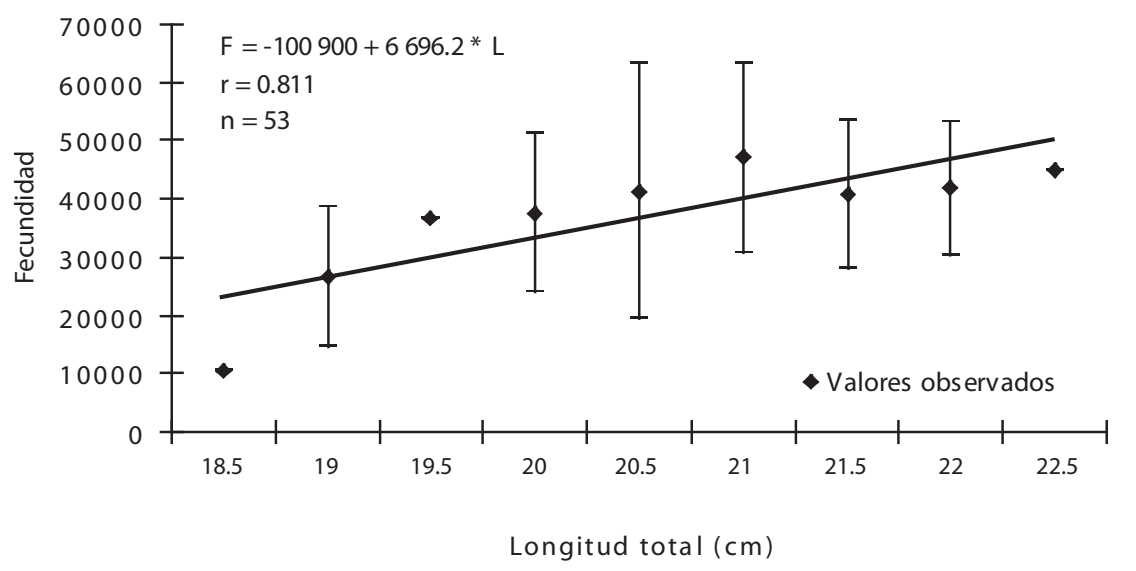

Fig. 6 Relación entre la fecundidad absoluta y la longitud total de Sardinella aurita del sureste de la Isla de Margarita.

Fig. 6 Relationship between length and absolute fecundity of Sardinella aurita of the south-eastern area of Margarita Island. 


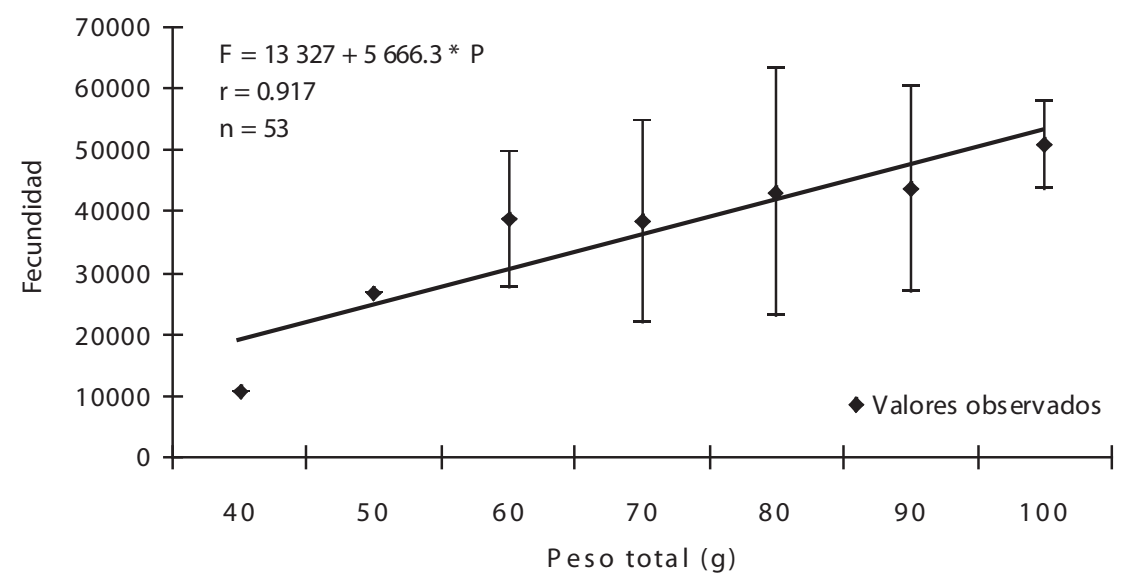

Fig. 7 Relación entre la fecundidad absoluta y el peso total de Sardinella aurita del sureste de la Isla de Margarita.

Fig. 7 Relationship between total weight and absolute fecundity of Sardinella aurita of the south-eastern area of Margarita Island.

motivados por factores ambientales (Margalef 1974), alteraciones en los ciclos anuales y variaciones entre subpoblaciones (Nikolsky 1963), o por el efecto de la presión de pesca sobre el recurso (González 1985, González y Eslava 2000).

En cuanto a la época de desove, la presencia de individuos desovados durante todos los meses de estudio, evidencia que la especie se reproduce durante todo el año. Sin embargo, tanto la frecuencia mensual de las fases de madurez sexual, como el índice de gónadas, señalan la existencia de dos periodos reproductivos de mayor intensidad (enero-marzo y octubrediciembre). Los mayores valores estimados del índice de gónadas se observaron entre enero y marzo (máximo desove), lo que determinaría un reclutamiento intenso en el segundo trimestre. González (2006) señala que el ingreso de nuevos reclutas al área de pesca ocurre, en los dos primeros trimestres del año, pero con mayor grado en el segundo trimestre, correspondiente a los meses de abril a junio que coincide con la época de vientos fuertes responsables de la surgencia costera, registrándose reclutamientos superiores a los 3300 millones de individuos.

Estos resultados coinciden con los observados en el nororiente de Venezuela por
Guzmán et al. (1999, 2001): enero-marzo y octubre-noviembre y Barrios (2002): febrero-marzo y octubre-noviembre; pero difieren con los obtenidos por Etchevers (1974): noviembre-junio, D'Suze (1981): mayo-junio y noviembre-febrero, Reyes (1981): mayo-junio y octubre-noviembre, Ramírez y Huq (1986): diciembre-mayo, Freón et al. (1997): noviembre-marzo y Mendialdúa (2004): abril-julio y noviembre, y Tsikliras y Antonopoulou (2006): mayo-julio en el Mar Egeo. La ocurrencia anual de dos periodos de desove con variaciones en tiempo y espacio pueden deberse a la estrategia reproductiva oportunista de $S$. aurita, que desova cuando las condiciones ambientales le son favorables para la sobrevivencia de sus huevos y larvas. Los valores máximos de desove pueden variar año tras año y estarían influenciados por las circunstancias climáticas asociados con épocas de alta productividad, como la surgencia costera y los aportes de los efluentes de ríos, como el Orinoco, en el periodo de lluvias (Frèon et al. 1997, González et al. 2007).

La talla calculada de madurez sexual de $\mathrm{L}_{50}$ igual a $20 \mathrm{~cm}$ fue semejante con la obtenida por Etchevers (1974) y Frèon et al. (1997), inferior a la determinada por Mendialdúa (2004): $20.29 \mathrm{~cm}$ y superior a las estimadas por Reyes 
(1981): 16 a 16.5 cm, Ramírez y Huq (1986): $16.9 \mathrm{~cm}$, Guzmán et al. (1999): $19.5 \mathrm{~cm}$ y Tsikliras y Antonopoulou (2006): 15.5 y 16.8 $\mathrm{cm}$ en machos y hembras del Mar Egeo, respectivamente. Tales diferencias provenientes de distintas zonas del oriente venezolano como de otras latitudes a través del tiempo, sugieren que esta talla varía no solo espacial, sino también temporalmente (Frèon et al. 1997).

La presencia de óvulos de diferente tamaño en los ovarios maduros, pone en evidencia el carácter parcial y continuo del desove de $S$. aurita al no madurar los óvulos al mismo tiempo, tal como lo afirman Figuera (1967), Reyes (1981), Ramírez y Huq (1986) y Guzmán et al. (2001). La fecundidad varió considerablemente, incluso entre ejemplares de talla y peso similares, como lo reportado en $S$. aurita del Mediterráneo (Tsikliras y Antonopoulou 2006). Estos valores se encontraron cercanos a los estimados para el Golfo de Cariaco por Figuera (1967) quien reporta de 9783 a 77 117 óvulos por hembra y para el nororiente de Venezuela por Reyes (1981) que encontró de 17720 a 60800 óvulos por hembra, Ramírez y Huq (1986) obtuvieron de 8659 a 39416 óvulos por hembra, Mendoza (1990) reportó de 9000 a 40000 óvulos por hembra y Guzmán et al. (1999) encontraron de 8456 a 30152 óvulos por hembra. Estas diferencias pueden estar relacionadas con factores ambientales y/o biológicos, ya que la fecundidad es altamente variable entre o en una misma especie, y puede cambiar entre años e incluso entre peces de una misma población, tamaño y edad (Tresierra y Culquichicón 1993), más aún si el tipo de desove es parcial (Guzmán et al. 1999), el cual influye notoriamente en la variabilidad del reclutamiento originando incertidumbre en los niveles de abundancia de la población de sardina (González 2006).

La fecundidad en $S$. aurita mostró proporcionalidad de manera consistente con el tamaño y peso corporal, resultados semejantes han sido reportados por otros autores (Figuera 1967, Reyes 1981, Ramírez y Huq 1986, Guzmán et al. 1999, Tsikliras y Antonopoulou 2006). Sin embargo, la relación entre la fecundidad y la talla no fue de tipo potencial, como es característico en la mayoría de los peces (Tresierra y Culquichicón 1993), sino de forma lineal, lo cual debe ser producto de la alta variabilidad de la fecundidad absoluta de individuos de longitud similar. Igualmente la fecundidad relativa osciló considerablemente entre individuos de talla y peso similares, ubicándose la media (519 óvulos/g) por debajo de los valores reportados por Reyes (1981): 681 óvulos/g para el nororiente de Venezuela y superior a los establecidos por Ramírez y Huq (1986): 222 óvulos/g para el Golfo de Cariaco y Tsikliras y Antonopoulou (2006): 462 óvulos/g para el Mediterráneo.

\section{AGRADECIMIENTOS}

Agradecemos a Darcy Romero por el suministro de las muestras biológicas, a Berta Parra profesora del Instituto Oceanográfico de Venezuela por su apoyo logístico en el desarrollo de la investigación, a Juan Ignacio Gaviria profesor de la Escuela de Ciencias Aplicadas del Mar por la revisión del manuscrito y a Francisco Guevara técnico pesquero del Instituto de Investigaciones Científicas por su colaboración en el trabajo de laboratorio. Al Consejo de Investigación de la Universidad de Oriente y al Fondo Nacional de Investigaciones Científicas y Tecnológicas por el financiamiento parcial del trabajo.

\section{RESUMEN}

Se determinó la proporción y madurez sexual, fecundidad y época de desove de la sardina, Sardinella aurita Valenciennes, 1847 del sureste de la Isla de Margarita, Venezuela; a través de muestreos mensuales provenientes de la pesca comercial desde enero 2004 hasta abril 2005, examinándose un total de 3736 individuos. La composición global por sexos fue de $55.06 \%$ hembras y $44.19 \%$ machos, siendo la proporción sexual 1:1.25 $\left(\chi^{2}=44.454\right.$, $\mathrm{p}<0.05)$. La talla de primera madurez sexual del $50 \%$ de la población fue de $20 \mathrm{~cm}$ de longitud. Se corroboró el carácter parcial y continuo del desove de la especie, cuyo periodo ocurrió durante todo el año, con dos periodos de relativa intensidad, correspondientes al primer (enero, febrero, marzo) y último trimestre (octubre, noviembre, diciembre) del año. La fecundidad absoluta osciló entre 
10530 a 83779 óvulos, y la relativa varió entre 149 a 1020 óvulos/g; siendo la fecundidad proporcional a la talla $(\mathrm{F}=-100900+6696.2 * \mathrm{~L})$ y al peso $(\mathrm{F}=13327+$ $5666.3 * \mathrm{P})$. Se concluye que la sardina presenta un potencial reproductivo alto y fluctuante, atribuida posiblemente, a la variabilidad ambiental.

Palabras clave: Sardinella aurita, reproducción, Isla de Margarita, Venezuela.

\section{REFERENCIAS}

Barrios, A. 2002. Periodicidad del reclutamiento y formación de cohortes juveniles en la sardina, Sardinella aurita Valenciennes, 1847 (Pisces: Clupeidae) en el Golfo de Cariaco, Estado Sucre, Venezuela. Tesis de Licenciatura, Universidad de Oriente, Cumaná, Sucre, Venezuela.

Cervigón, F. 1991. Los Peces Marinos de Venezuela. Volumen I, Fundación Científica de Los Roques. Caracas, Venezuela.

D`Suze, G. 1981. Aspectos de la energética reproductiva del pez migratorio Sardinella aurita Clupeidae. Tesis de Licenciatura, Universidad de Oriente, Cumaná, Sucre, Venezuela.

Eslava, N. 2007. Alimentación y Reproducción de Peces. 2da Edición, Universidad de Oriente. Universitaria. Cumaná, Sucre, Venezuela.

Etchevers, S. 1974. Variaciones morfométrico-merísticas, biología y tamaño del stock de sardina en el Nororiente de Venezuela. Bol. Inst. Oceanog. Ven. 15: 119-132.

Figuera, F. 1967. Contribución a los conocimientos de la fecundidad en la Sardinella sp. del Golfo de Cariaco. Tesis de Licenciatura, Universidad de Oriente, Cumaná, Sucre, Venezuela.

Fischer, W. (ed). 1977. Species identification sheets for fishery purposes western central Atlantic. Marine Resources Service. FAO. Vol II. Roma, Italia.

Fréon, P., M. El Khattabi, J. Mendoza \& R. Guzmán. 1997. Unexpected reproductive strategy of Sardinella aurita of the coast of Venezuela. Mar. Biol. 128: 363-372.

Gaertner, D. \& F. Laloé. 1986. Etude biométrique de la taille á première maturité sexuelle de Geryon maritae Manning et Holthuis, 1981 du Sènegàl. Oceanol. Acta. 9: 479-487.

González, L. W. 1985. Determinación de edad y crecimiento de la sardina, Sardinella aurita Valenciennes, 1847 (Pisces: Clupeidae) de la región nororiental de Venezuela. Bol. Inst. Oceanog. Ven. 24: 111-128.
González, L. W. \& N. Eslava. 2000. Crecimiento y mortalidad natural de la sardina, Sardinella aurita (Teleostei: Clupeidae) del Estado Nueva Esparta, Venezuela. Rev. Biol. Mar. Oceanog. 35: 83-91.

González, L. W. 2006. Análisis de la pesquería artesanal de la sardina (Sardinella aurita) del estado Nueva Esparta, Venezuela: un enfoque bioeconómico precautorio. Tesis de Doctorado, Cinvestav, Mérida, Yucatán, México.

González, L. W., J. Euán, N. Eslava \& J. Suniaga. 2007. La pesca de sardina, Sardinella aurita (Teleostei: Clupeidae) asociada a la variabilidad ambiental del ecosistema de surgencia costera de Nueva Esparta, Venezuela. Rev. Biol. Trop. 55: 279-286.

Guzmán, R., G. Gómez \& M. Penott. 1998. Aspectos biológicos y pesquería de la sardina (Sardinella aurita) en Golfo de Cariaco, Venezuela. Zoo. Trop. 16: 149-162.

Guzmán, R. \& M. Penott. 1999. Monitoreo del recurso sardina, Sardinella aurita en las costas de la Península de Araya. Bol. Inst. Oceanog. Ven. 38: 37-38.

Guzmán, R., G. Gómez, M. Penott \& G. Vizcaino. 1999. Estructura de tallas y reproducción de la sardina Sardinella aurita en el nororiente de Venezuela. Zoo. Trop. 17: 155-174.

Guzmán, R., F. Arocha \& G. Gómez. 2001. Tipo de desove de la sardina (Sardinella aurita Valenciennes, 1847) en el nororiente de Venezuela: Resultados preliminares. Zoo. Trop. 19: 173-183.

Heald, E. \& R. Griffiths. 1967. La determinación por medio de la lectura de escamas de la sardina, Sardinella anchovia, del Golfo de Cariaco, Venezuela Oriental. Serie Recursos y Explotación Pesqueros. Ministerio de Agricultura y Cría. 1: 375-446.

Holden, M. \& D. Raitt. 1975. Manual de ciencia pesquera. Parte 2. Métodos para investigar los recursos y su aplicación. Documento técnico de pesca, FAO. $\mathrm{N}^{\mathrm{o}}$ 115. Roma, Italia.

Hoydal, K., C. Rorvik \& P. Sparre. 1982. Estimation of effective mesh sizes and their utilization in assessment. Dana. 2: 69-95.

Margalef, R. 1974. Ecología. Omega. Barcelona, España.

Mendialdua, J. 2004. Aspectos reproductivos de la sardina (Sardinella aurita) del sureste de la Isla de Margarita, Estado Nueva Esparta, Venezuela. Tesis de Licenciatura, Universidad de Oriente, Boca de Río, Venezuela. 
Mendoza, J. 1990. La pesquería de sardina en el nororiente venezolano: evaluación y perspectiva de desarrollo. Resultados de Talleres sobre la pesca en Venezuela. Ministerio de Agricultura y Cría, Caracas, Venezuela.

Nikolsky, G. 1963. The ecology of fishes. Academic London. Nueva York, EEUU.

Palazón, J. 2007. Reproduction of the white grunt, Haemulon plumieri (Lacépede, 1802) (Pisces: Haemulidae) from Margarita Island, Venezuela. Sci. Mar. 71: 429-440.

Parker, K. 1985. Biomass model for the egg production method, p. 5-6. En R. Lasker (ed.). An egg production method for estimating spawning biomass of pelagic fish: Application to the Northern Anchovy, Engraulis mordax. National Oceanic and Atmospheric Administration (NOAA). Tech. Report. 36.

Ramírez, I. \& M. Huq. 1986. Aspectos reproductivos de la sardina, Sardinella aurita Valenciennes, 1847 (Pisces: Clupeidae) del Golfo de Cariaco, Estado Sucre, Venezuela. Bol. Inst. Oceanog. Ven. 25: 3-20.
Reyes, D. 1981. Aspectos reproductivos de Sardinella aurita (Pisces: Clupeidae). Tesis de Licenciatura, Universidad de Oriente, Cumaná, Sucre, Venezuela.

Ricker, W. 1973. Linear regressions in fishery research. J. Fish. Res. Bd. Can. 30: 409-434.

Schaefer, M. \& C. Orange. 1956. Studies on the sexual development and spawning of yellow-fin tuna (Neothunnus macropterus) and skipjack (Katsuwonus pelamis) in three areas of the eastern Pacific Ocean by examination of gonads. Inter. Tun. Comm. Bull. 1: 281-349.

Steel, R. \& J. Torrie. 1985. Bioestadística: Principios y procedimientos. Segunda Edición. Mc. Graw-Hill, Latinoamericana S.A. Bogotá, Colombia.

Tresierra, A. \& M. Culquichicón. 1993. Biología Pesquera. Libertad. Trujillo, Perú.

Tsikliras, A. \& E. Antonopoulou. 2006. Reproductive biology of round sardinella (Sardinella aurita) in the north-eastern Mediterranean. Sci. Mar. 70: 281-290. 